\title{
EQUIVARIANT CHAIN COMPLEXES, TWISTED HOMOLOGY AND RELATIVE MINIMALITY OF ARRANGEMENTS
}

\author{
By ALEXANDRU DIMCA AND ŞTEFAN PAPADIMA ${ }^{1}$
}

ABStraCT. - We show that the $\pi$-equivariant chain complex $\left(\pi=\pi_{1}(M(\mathcal{A}))\right), C_{\bullet}(\tilde{X})$, associated to a Morse-theoretic minimal $C W$-structure $X$ on the complement $M(\mathcal{A})$ of an arrangement $\mathcal{A}$, is independent of $X$. The same holds for all scalar extensions, $C \bullet(\widetilde{X}) \otimes_{\mathbb{Z} \pi} \mathbb{K} \mathbb{Z}, \mathbb{K}$ a field, where $X$ is an arbitrary minimal $C W$-structure on a space $M$. When $\mathcal{A}$ is a section of another arrangement $\widehat{\mathcal{A}}$, we show that divisibility properties of the first Betti number of the Milnor fiber of $\mathcal{A}$ obstruct the homotopy realization of $M(\mathcal{A})$ as a subcomplex of a minimal structure on $M(\widehat{\mathcal{A}})$.

If $\widehat{\mathcal{A}}$ is aspherical and $\mathcal{A}$ is a sufficiently generic section of $\widehat{\mathcal{A}}$, then $H_{*}(M(\mathcal{A}) ; L)$ may be described in terms of $\pi, L$ and $\chi(M(\mathcal{A}))$, for an arbitrary local system $L$; explicit computations may be done, when $\widehat{\mathcal{A}}$ is fiber-type. In this case, explicit $\mathbb{K} \mathbb{Z}$-presentations of arbitrary abelian scalar extensions of the first nontrivial higher homotopy group of $M(\mathcal{A}), \pi_{p}(M)$, may also be obtained. For nonresonant abelian scalar extensions, the $\mathbb{C} \mathbb{Z}$-rank of $\pi_{p}(M) \otimes_{\mathbb{Z} \pi} \mathbb{C} \mathbb{Z}$ is combinatorially determined.

(C) 2004 Elsevier SAS

RÉSUMÉ. - Nous montrons que le complexe de chaînes $C_{\bullet}(\widetilde{X})$ associé à une structure cellulaire minimale de Morse, $X$ sur le complémentaire $M(\mathcal{A})$ d'un arrangement $\mathcal{A}$, est indépendant de $X$ en tant que $\mathbb{Z} \pi$-complexe, où $\pi=\pi_{1}(M(\mathcal{A}))$. Le même résultat reste vrai pour toutes les extensions scalaires $C \bullet(\widetilde{X}) \otimes_{\mathbb{Z} \pi} \mathbb{K} \mathbb{Z}, \mathbb{K}$ étant un corps et $X$ une structure minimale sur un espace $M$.

Lorsque $\mathcal{A}$ s'obtient comme une section d'un autre arrangement $\widehat{\mathcal{A}}$, nous montrons que les propriétés de divisibilité du premier nombre de Betti de la fibre de Milnor associée à $\mathcal{A}$ donnent des obstructions à la réalisation de $M(\mathcal{A})$ comme un sous-complexe d'une structure minimale sur $M(\widehat{\mathcal{A}})$. Si $\widehat{\mathcal{A}}$ est asphérique et $\mathcal{A}$ en est une section suffisamment générale, alors $H_{*}(M(\mathcal{A}) ; L)$, pour $L$ un système local quelconque, peut être décrit en fonction de $\pi, L$ et $\chi(M(\mathcal{A}))$. Des calculs explicites sont possibles lorsque $\widehat{\mathcal{A}}$ est de type fibré. Dans cette situation, des présentations explicites pour des extensions scalaires abéliennes de $\pi_{p}(M)$, le premier groupe d'homotopie supérieur non-nul de $M(\mathcal{A})$, peuvent aussi être obtenues. Pour les extensions scalaires abéliennes qui sont non-résonantes, le $\mathbb{C} \mathbb{Z}$-rang de $\pi_{p}(M) \otimes_{\mathbb{Z} \pi} \mathbb{C} \mathbb{Z}$ est déterminé par la combinatoire.

(c) 2004 Elsevier SAS

\section{Introduction}

Let $\mathcal{A}=\left\{H_{0}, H_{1}, \ldots, H_{n}\right\}$ be a complex hyperplane arrangement in $\mathbb{P}^{r-1}$, with complement $M=M(\mathcal{A})=\mathbb{P}^{r-1} \backslash \bigcup_{i=0}^{n} H_{i}$, and fundamental group $\pi=\pi_{1}(M)$. The cohomology ring of the complement, with arbitrary constant coefficients, was computed by Orlik and Solomon [20];

\footnotetext{
${ }^{1}$ Partially supported by grant CNCSIS 693/2002 of the Romanian Ministry of Education and Research. 
their description involves solely the combinatorics of $\mathcal{A}$, that is, the associated intersection lattice $\mathcal{L}(\mathcal{A})$. The fundamental group $\pi$ is complicated in general, but nevertheless rather well understood and accessible to concrete computations, see for instance $[5,17]$. Note however that $\mathcal{L}(\mathcal{A})$ does not determine $\pi$ in general, as the example of Rybnikov [24] shows.

Much less is known about the homology groups $H_{*}(M ; L)$ of $M$ with twisted coefficients (alias $\mathbb{Z} \pi$-modules), $L$. Our aim in this paper is to get more insight on such groups $H_{*}(M ; L)$, both at the general and computational level, along the lines sketched in our previous work $[8$, Remark 12(ii)].

\subsection{Absolute minimality and equivariant chain complexes}

Among other things, we have proved in [8] that $M$ has the homotopy type of a minimal $C W$-complex $X$, that is, of a $C W$-complex with trivial cellular incidences (a result independently obtained by Randell [23]). In this paper, we go further and analyze in Section 2 the associated $\mathbb{Z} \pi$-chain complex $C_{\bullet}(\widetilde{X})$ of the universal cover $\widetilde{X}$ of $X$. As it is well known [26], this is the universal object encoding twisted homology information, since

$$
H_{*}(M ; L)=H_{*}\left(C \bullet(\widetilde{X}) \otimes_{\mathbb{Z} \pi} L\right), \quad \text { for all } L .
$$

At the same time, this is also relevant for computations related to higher homotopy groups (a very difficult subject, in general), since

$$
\pi_{p}(M)=H_{p}(C \bullet(\widetilde{X}))
$$

by Hurewicz, where $\pi_{p}(M)$ is the first higher non-trivial homotopy group of $M$ (for $M$ nonaspherical).

Note that the $\mathbb{Z} \pi$-chain complex $C \bullet(\widetilde{X})$ may depend on the choice of $C W$-structure on $M$. In general, all one can say is that the $\mathbb{Z} \pi$-chain homotopy type is independent of $X$. In this direction, we have two general results where we can upgrade the above independence property to chain isomorphism type.

The first one is Corollary 2.6, which says that the $\mathbb{Z} \pi$-chain complex $C_{\bullet}(\tilde{X})$ actually defines a new invariant of $\mathcal{A}$, depending only on the projective equivalence type of $\mathcal{A}$. Key to our proof is an argument involving Whitney stratifications and transversality conditions for projective flags. In this way, we recover in particular a series of results, proved by Cohen and Orlik in [1] and [3].

A standard technique in topology is abelianization (leading for instance to the theory of Alexander invariants, see [14]). When a $\mathbb{Z} \pi$-module like $\pi_{p}(M)$ in (1.2) above looks intractable, it is generally hoped that $\pi_{p}(M) \otimes_{\mathbb{Z} \pi} \mathbb{K} \mathbb{Z}$ will be more manageable, where $\mathbb{K}$ is a field and

$$
\nu: \pi \rightarrow \mathbb{Z}
$$

is a group character, inducing a change of rings, $\nu: \mathbb{Z} \pi \rightarrow \mathbb{K} \mathbb{Z}$. In this way we pass from modules over a very bad ring, namely $\mathbb{Z} \pi$, to modules over the principal ideal domain $\mathbb{K} \mathbb{Z}$, where we have structure theorems for the finite type modules. The abelian extensions of scalars $\pi_{p}(M) \otimes_{\mathbb{Z} \pi} \mathbb{K} \mathbb{Z}$ obtained in this way will be called character-abelianizations.

Our second main result in Section 2, Theorem 2.9, says that the $\mathbb{K} \mathbb{Z}$-chain complex $C .(\widetilde{X}) \otimes_{\mathbb{Z} \pi} \mathbb{K} \mathbb{Z}$ is independent of the minimal $C W$-structure $X$ on $M$, for an arbitrary space $M$.

\subsection{General position and cell structures}

Now assume that $\mathcal{A}$ is a $k$-generic section of another arrangement, $\widehat{\mathcal{A}}$ in $\mathbb{P}^{m-1}$. By ' $k$-genericity' we mean a set of transversality conditions, depending on $k$, with respect to certain 
strata of the natural stratification of $\mathbb{P}^{m-1}$ associated to $\widehat{\mathcal{A}}$; see $[8,(1)]$ for details. When we speak about $\widehat{\mathcal{A}}$-genericity, we simply mean that $\mathbb{P}^{r-1}$ (regarded as a linear subspace in $\mathbb{P}^{m-1}$ ) is transverse to all $\widehat{\mathcal{A}}$-strata.

Following the program from $[8, \S \S 5-6]$, our idea here is to get from $M(\widehat{\mathcal{A}})$ twisted homology information on $M(\mathcal{A})$, assuming $k$-genericity. The key tool is provided by [8, Proposition 14], which says that one may replace, up to homotopy, the inclusion $M(\mathcal{A}) \hookrightarrow M(\widehat{\mathcal{A}})$ by a cellular map, $f: X \rightarrow Y$, between minimal $C W$-complexes, such that

$$
f_{\mid X^{(k)}}=\mathrm{id}
$$

When $M(\widehat{\mathcal{A}})$ is aspherical and $k \geqslant 2$, it follows from (1.4) above that $M(\widehat{\mathcal{A}})$ is a $K(\pi, 1)$. Moreover, Proposition 15 from [8] implies that one may replace in (1.4) $k$ by $p=p(M(\mathcal{A})$ ), where $p$ is a homotopy invariant of $M(\mathcal{A})$, introduced in [22].

Assuming full $\widehat{\mathcal{A}}$-genericity, (1.4) improves to

$$
X=Y^{(r-1)},
$$

where $f$ is the inclusion of the $(r-1)$-skeleton, $Y^{(r-1)} \hookrightarrow Y$. The basic example when the homotopy formula (1.5) holds is provided by Boolean genericity (in other words, usual general position); see Hattori's pioneering paper [13]. This generalizes to fiber-type genericity. The arrangements $\mathcal{A}$ which are $\widehat{\mathcal{A}}$-generic sections of fiber-type arrangements $\widehat{\mathcal{A}}$ may be defined in purely combinatorial terms: they are the hypersolvable arrangements (introduced in [15]) for which $p=r-1$; see Section 4.4.

\subsection{Relative minimality and twisted homology}

In Section 3 (Theorem 3.6 and Example 3.7) we prove that, in general, the homotopy formula (1.4) from Section 1.2 is the best possible result. In spite of some homological and Morse-theoretic positive evidence, it turns out that the ideal relative minimality property,

$$
f=\text { subcomplex inclusion, }
$$

cannot hold in full generality, not even on $X^{(k+1)}$.

To prove this, we explore the consequences of (1.6) on the first Betti number of the Milnor fiber of $\mathcal{A}$. This in turn uses a decomposition of the homology of the Milnor fiber, see e.g. Cohen and Suciu in [4], in terms of the homology of $M(\mathcal{A})$ with certain twisted coefficients.

\subsection{Fiber-type arrangements and free differential calculus}

Most results on twisted homology of arrangements available in the literature assume nonresonant coefficients and take the form of vanishing theorems. There is however a particularly remarkable exception, due to Cohen and Suciu [6], from the computational point of view.

The authors of [6] consider finitely-presented groups, $\pi$, which are iterated semidirect products of free groups, with all monodromy actions trivial on homology. They use the Fox free differential calculus to construct a minimal $C W$-structure $Y$ on $K(\pi, 1)$, and to describe explicitly the associated $\mathbb{Z} \pi$-chain complex, $C_{\bullet}(\tilde{Y})$.

On the one hand, this leads to explicit twisted homology computations with non-trivial result; see $[4,6]$. On the other hand, this has implications in arrangement theory, coming from the fact that the fundamental groups of fiber-type arrangements all have such a semidirect product structure; see Falk and Randell [10]. 


\subsection{Twisted homology with resonant coefficients}

We come back, in Section 4, to our framework, explained in Section 1.2. Let $\mathcal{A}$ be a sufficiently generic section of an aspherical arrangement $\widehat{\mathcal{A}}$, with complement $M=M(\mathcal{A})$ and fundamental group $\pi=\pi_{1}(M)$. Set $p=p(M)$. Let $L$ be a resonant (that is, arbitrary) $\mathbb{Z} \pi$-module.

Using (1.4), we infer in Theorem 4.2 that

$$
H_{<p}(M ; L)=\operatorname{Tor}_{<p}^{\mathbb{Z} \pi}(\mathbb{Z}, L) .
$$

When $\widehat{\mathcal{A}}$ is fiber-type, the $\operatorname{Tor}^{\mathbb{Z} \pi}$-groups from (1.7) above may be explicitly computed by Fox calculus, see Section 1.4, even though $\mathcal{A}$ is no longer of fiber type when $r<m$. Assuming only that there are no collinearity relations among the defining equations of the hyperplanes of $\mathcal{A}$, Theorem 4.2 applies and everything becomes extremely simple: $\pi=\mathbb{Z}^{n}$, and the Fox resolution is the standard Koszul resolution of $\mathbb{Z}$ over $\mathbb{Z} \mathbb{Z}^{n}$; see Corollary 4.3.

In Theorem 4.5, we assume full $\widehat{\mathcal{A}}$-genericity, hence the stronger homotopy formula (1.5) becomes available. Via an Euler characteristic argument, we are thus able to extend (1.7) above to a full computation of $H_{*}(M ; L)$, involving only $\operatorname{Tor}_{*}^{\mathbb{Z} \pi}(\mathbb{Z}, L)$ and $\chi(M)$.

\subsection{Character-abelianizations of higher homotopy groups}

Very little is known about higher homotopy groups of complements in $\mathbb{P}^{r-1}$ of complex projective hypersurfaces, $V(h)=\{h=0\}$. Let $H$ be a hyperplane in $\mathbb{P}^{r-1}$, generic with respect to $V(h)$. Set $M=\mathbb{P}^{r-1} \backslash(V(h) \cup H)$, and $\pi=\pi_{1}(M)$. When $h$ is irreducible, Libgober [18] showed that, under certain additional hypotheses, $\pi=\mathbb{Z}$, and $\pi_{p}(M)$ (the first higher non-trivial homotopy group of $M$ ) is a torsion $\mathbb{Q} \mathbb{Z}$-module.

Arrangement complements are the simplest non-irreducible examples. Here, $\pi$ is much more complicated, and character-abelianizations of $\pi_{p}(M)$ may have strictly positive $\mathbb{Q Z Z}$-rank; see our Theorem 5.3(3) from Section 5.

Our results on character-abelianizations of $\pi_{p}(M)$ assume full $\widehat{\mathcal{A}}$-genericity, like in Theorem 4.5 (see Section 1.5). The reason is that in this case the minimal $\mathbb{Z} \pi$-resolution of $\pi_{p}(M)$ from [8, Theorem 18(ii)] is available.

In Theorem 5.6, we give a formula for the $\mathbb{K} \mathbb{Z}$-rank of an arbitrary character-abelianization of $\pi_{p}(M)$, involving $\operatorname{Tor}_{*}^{\mathbb{Z} \pi}(\mathbb{Z}, \mathbb{K} \mathbb{Z})$ and $\chi(M)$.

Assume now moreover that $\widehat{\mathcal{A}}$ is fiber-type. Then Corollary 2.11 provides an explicit $\mathbb{K} \mathbb{Z}$-presentation of an arbitrary character-abelianization, $\pi_{p}(M) \otimes_{\mathbb{Z} \pi} \mathbb{K} \mathbb{Z}$. In this case, Theorem 5.3 also provides an explicit combinatorial formula, for the $\mathbb{C} \mathbb{Z}$-rank of a so-called $\widehat{\mathcal{A}}$-nonresonant character-abelianization (see Definition 5.2) of $\pi_{p}(M)$. The last result represents a new combinatorial determination phenomenon, related to higher homotopy groups of arrangements, to be compared with [8, Theorem 23].

\section{Minimal equivariant chain complexes}

\subsection{Generic flags and minimal $C W$-structures}

Let $\mathcal{A}$ be a complex hyperplane arrangement in $\mathbb{P}(U)$, with complement $M:=M(\mathcal{A})$ and fundamental group $\pi:=\pi_{1}(M)$. In Section 4 from [8], we have constructed a minimal $C W$-structure on $M$, that is, a $C W$-complex $X$ homotopy equivalent to $M$, having as many $k$-cells as the $k$ th Betti number, for all $k$. Our construction involved various choices. To examine 
this issue, we will need several definitions. Without any loss of generality, we may assume that $\mathcal{A}$ is essential; see [21, p. 197].

The intersection lattice, $\mathcal{L}(\mathcal{A})$, is the set of edges of $\mathcal{A}$ (that is, the nonempty intersections of hyperplanes from $\mathcal{A}$ ), ordered by reverse inclusion. One has a canonical stratification of $\mathbb{P}(U)$, $\mathcal{S}(\mathcal{A}):=\left\{\mathcal{S}_{S}\right\}_{S \in \mathcal{L}(\mathcal{A})}$, with the property that $\overline{\mathcal{S}_{S}}=S$, for all $S$; see [12, III.3.1 and III.4.5]. Set $r:=\operatorname{dim} U$.

DEFINITION 2.2. - An increasing projective flag in $\mathbb{P}(U), \mathcal{F}=\left\{\mathcal{F}_{s}\right\}_{0 \leqslant s<r}$ (where $\operatorname{dim} \mathcal{F}_{s}=s$, for all $s$ ) is called $\mathcal{A}$-generic if $\mathcal{F}_{s}$ is transverse to $\mathcal{S}\left(\mathcal{A}^{\mathcal{F}_{s+1}}\right)$, for $s<r-1$, where $\mathcal{A}^{\mathcal{F}_{s+1}}$ denotes the projective arrangement in $\mathcal{F}_{s+1}$ obtained by restriction from $\mathcal{A}$.

An $\mathcal{A}$-generic flag gives a finite increasing filtration of $M,\left\{F_{s} M\right\}_{0 \leqslant s<r}$ :

$$
F_{s} M:=M \cap \mathcal{F}_{s}
$$

Definition 2.3. - Let $X$ be an $(r-1)$-dimensional minimal complex. A homotopy equivalence, $\varphi: X \rightarrow M$, is compatible with a given $\mathcal{A}$-generic flag if $\varphi$ restricts to homotopy equivalences, $\varphi_{s}: X^{(s)} \rightarrow F_{s} M$, for $0 \leqslant s<r$.

Our (absolute) minimality result from [8, Section 4] reads then: for any $\mathcal{A}$-generic flag, $\mathcal{F}$, there exists an $\mathcal{F}$-compatible minimal structure on $M(\mathcal{A}), X$, in the sense of the above Definition 2.3.

Let now $\left(Z, z_{0}\right)$ be a reasonable based space (e.g., a connected complex, or a connected manifold), endowed with a finite increasing filtration, $\left\{F_{s} Z\right\}_{s \geqslant 0}$, such that $F_{0} Z=\left\{z_{0}\right\}$. Let $p_{Z}:\left(\widetilde{Z}, \tilde{z_{0}}\right) \rightarrow\left(Z, z_{0}\right)$ be a universal cover. Set $\pi:=\pi_{1}\left(Z, z_{0}\right)$. Define

$$
F_{s} \widetilde{Z}:=p_{Z}^{-1}\left(F_{s} Z\right), \quad \text { for } s \geqslant 0 .
$$

The associated $\pi$-equivariant chain complex,

$$
C_{\bullet}(\widetilde{Z}):=\left\{d_{q}: H_{q}\left(F_{q} \widetilde{Z}, F_{q-1} \widetilde{Z}\right) \rightarrow H_{q-1}\left(F_{q-1} \widetilde{Z}, F_{q-2} \widetilde{Z}\right)\right\}_{q},
$$

is endowed with the boundary maps, $d_{q}$, coming from the triple $\left(F_{q}, F_{q-1}, F_{q-2}\right)$, in the standard way. It is a chain complex of (right) $\mathbb{Z} \pi$-modules.

We are going to show that the (minimal) $\pi$-equivariant chain complex, $C_{\bullet}(\widetilde{X})$, from [8], is a well-defined invariant of $\mathcal{A}$. We do this in two steps.

Let $\varphi: X \rightarrow M$ be an $\mathcal{F}$-compatible homotopy equivalence, as in Definition 2.3. Denote by $\Phi$ the lift of $\varphi$ to (based) universal covers. Use $\varphi_{\#}: \pi_{1}(X) \stackrel{\sim}{\rightarrow} \pi_{1}(M)$ to identify fundamental groups. Denote by

$$
\tilde{\varphi}:=\left\{\widetilde{\varphi_{q}}: C_{q}(\widetilde{X}) \rightarrow C_{q}(\widetilde{M})\right\}_{q}
$$

the $\mathbb{Z} \pi$-chain map, induced by $\Phi$, between the equivariant chain complexes associated to the skeletal filtration on $X$, and the $\mathcal{F}$-filtration (2.1) on $M$ respectively.

LEMMA 2.4. - The above map (2.3) is an isomorphism of $\mathbb{Z} \pi$-chain complexes.

Proof. - By standard homotopy properties of fibrations, $p_{X}:\left(\widetilde{X}, F_{q} \widetilde{X}\right) \rightarrow\left(X, F_{q} X\right)$ will induce isomorphisms on all homotopy groups, for $q \geqslant 0$, and likewise for $M$. It follows that $\Phi:\left(\widetilde{X}, F_{q} \widetilde{X}\right) \rightarrow\left(\widetilde{M}, F_{q} \widetilde{M}\right)$ (and therefore $\Phi: F_{q} \widetilde{X} \rightarrow F_{q} \widetilde{M}$ ) induces isomorphisms on all homotopy groups, since $\varphi$ induces homotopy equivalences, $\varphi_{q}: F_{q} X \rightarrow F_{q} M$. By Whitehead's theorem, $\Phi:\left(F_{q} \widetilde{X}, F_{q-1} \widetilde{X}\right) \rightarrow\left(F_{q} \widetilde{M}, F_{q-1} \widetilde{M}\right)$ induces homology isomorphisms, for all $q$. 
This shows that the skeletal equivariant chain complex does not depend on the choice of $\mathcal{F}$-compatible minimal structure, for a fixed $\mathcal{A}$-generic flag, $\mathcal{F}$.

Lemma 2.5. - Let $\mathcal{F}$ and $\mathcal{F}^{\prime}$ be two $\mathcal{A}$-generic flags, with associated filtrations $F_{\bullet} M$ and $F_{\bullet}^{\prime} M$ respectively. There is a filtered homeomorphism,

$$
\Psi:\left(M, F_{\bullet} M\right) \stackrel{\sim}{\longrightarrow}\left(M, F_{\bullet}^{\prime} M\right) .
$$

Proof. - The set $\operatorname{Flag}(\mathbb{P}(U))$ of all the flags

$$
\mathcal{F}: \emptyset=\mathcal{F}_{-1} \subset \mathcal{F}_{0} \subset \cdots \subset \mathcal{F}_{r-1}
$$

in $\mathbb{P}(U)$ is a complex algebraic variety which is compact, smooth and connected, hence irreducible. The subset $F \operatorname{lag}(\mathcal{A}) \subset \operatorname{Flag}(\mathbb{P}(U))$ of all the $\mathcal{A}$-generic flags is an open Zariski subset in this irreducible variety, and as such it is connected. Consider the second projection

$$
p_{2}: \mathbb{P}(U) \times \operatorname{Flag}(\mathcal{A}) \rightarrow \operatorname{Flag}(\mathcal{A}) .
$$

On the product $\mathbb{P}(U) \times F \operatorname{lag}(\mathcal{A})$ there are two natural Whitney regular stratifications.

The first one, denoted by $\mathcal{X}$, is the product of the stratification $\mathcal{S}(\mathcal{A})$ on $\mathbb{P}(U)$ by the trivial stratification on $\operatorname{Flag}(\mathcal{A})$, i.e. $\mathcal{X}$ has as strata the products of the form

$$
X_{S}=\mathcal{S}_{S} \times \operatorname{Flag}(\mathcal{A})
$$

for $S \in \mathcal{L}(\mathcal{A})$, see [11, p. 12].

The second stratification, denoted by $\mathcal{Y}$, has as strata the following (constructible) submanifolds in $\mathbb{P}(U) \times F \operatorname{lag}(\mathcal{A})$ :

$$
Y_{j}=\left\{(x, \mathcal{F}) \in \mathbb{P}(U) \times \operatorname{Flag}(\mathcal{A}) ; x \in \mathcal{F}_{j} \backslash \mathcal{F}_{j-1}\right\}
$$

for $j=0,1, \ldots, r-1$.

To see that the stratification $\mathcal{Y}$ is Whitney regular, note that it is induced by the stratification $\mathcal{Z}$ on $\mathbb{P}(U) \times$ Flag $(\mathbb{P}(U))$ given by the orbits of the regular action

$$
\operatorname{Aut}(U) \times(\mathbb{P}(U) \times \operatorname{Flag}(\mathbb{P}(U))) \rightarrow \mathbb{P}(U) \times \operatorname{Flag}(\mathbb{P}(U))
$$

where $g \cdot(x, \mathcal{F})=(g x, g \mathcal{F})$. Indeed, the orbits of this action are exactly the sets

$$
Z_{j}=\left\{(x, \mathcal{F}) \in \mathbb{P}(U) \times \operatorname{Flag}(\mathbb{P}(U)) ; x \in \mathcal{F}_{j} \backslash \mathcal{F}_{j-1}\right\}
$$

for $j=0,1, \ldots, r-1$. The resulting stratification $\mathcal{Z}$ is Whitney regular by a general result on regular actions with finitely many orbits, see [11, p. 21].

Using the definition of an $\mathcal{A}$-generic flag, it follows that the two stratifications $\mathcal{X}$ and $\mathcal{Y}$ are transversal (or in general position) and hence their intersection $\mathcal{T}$ is also a Whitney regular stratification, see [11, p. 12]. Note that the strata of this stratification are all the non-empty intersections $T_{S, j}=X_{S} \cap Y_{j}$, in particular these intersections are connected. It can be shown by a direct computation that the restrictions

$$
p_{2}: T_{S, j} \rightarrow F \operatorname{Flag}(\mathcal{A})
$$


are all submersive surjections. Applying Thom's First Isotopy Lemma, see Theorem (5.2), [11, p. 58], to the second projection $p_{2}$ we get that $\mathbb{P}(U) \times \operatorname{Flag}(\mathcal{A})$ fibers over $\operatorname{Flag}(\mathcal{A})$ in the stratified sense. This means that for any two fibers of $p_{2}$, corresponding to two $\mathcal{A}$-generic flags $\mathcal{F}$ and $\mathcal{F}^{\prime}$, there is a homeomorphism $\mathbb{P}(U) \times\{\mathcal{F}\} \rightarrow \mathbb{P}(U) \times\left\{\mathcal{F}^{\prime}\right\}$ sending the strata of the stratifications induced by $\mathcal{T}$ into each other. This proves our claim by considering only the strata contained in $M$.

Corollary 2.6. - The $\mathbb{Z} \pi$-chain complex associated to any Morse-theoretic minimal $C W$-structure, $X$, on $M(\mathcal{A})$, constructed in [8], is an invariant of $\mathcal{A}$. Actually, the $\mathbb{Z} \pi$-chain complex $C_{\bullet}(\widetilde{X})$ depends only on the projective equivalence type of $\mathcal{A}$.

\subsection{Relation to work by Cohen-Orlik}

The $\pi$-equivariant chain complex from Corollary 2.6 is a potentially powerful invariant of the arrangement $\mathcal{A}$. This is due for instance to the fact that the first nonzero homology group of $C \bullet(\widetilde{X})$ is isomorphic to the first nonzero higher homotopy group of $M(\mathcal{A})$, when $M(\mathcal{A})$ is not aspherical; see $[8, \S \S 5-6]$, for various results on higher homotopy groups, inspired from this remark. This is also due to the fact that $C_{\bullet}(\widetilde{X})$ is the universal chain complex computing the twisted (co)homology of the complement. Indeed, let $N$ be an arbitrary left $\mathbb{Z} \pi$-module. Then

$$
H_{*}(M(\mathcal{A}) ; N)=H_{*}\left(C \bullet(\tilde{X}) \otimes_{\mathbb{Z} \pi} N\right)
$$

(and similarly for cohomology); see [26, Chapter VI].

From basic equation (2.4), we may easily recover (in homological form) various results, found by D. Cohen in [1] for the case of a $\mathbb{C}$-vector space $N$, and then reformulated in terms of flags by D. Cohen and P. Orlik [3]. Note that the flags $\mathcal{F}$ used in [3, §2.3] coincide with our $\mathcal{A}$-generic flags from Definition 2.2.

If $\left(Z, z_{0}\right)$ is a reasonable filtered space, as in Section 2.1, and $N$ is a left $\mathbb{Z} \pi_{1}\left(Z, z_{0}\right)$-module, one may consider the chain complex

$$
C_{\bullet}(Z ; N):=\left\{d_{q}: H_{q}\left(F_{q} Z, F_{q-1} Z ; N\right) \rightarrow H_{q-1}\left(F_{q-1} Z, F_{q-2} Z ; N\right)\right\}_{q},
$$

(the twisted version of (2.2)). If $\varphi: X \rightarrow M$ is an $\mathcal{F}$-compatible homotopy equivalence, as in Definition 2.3, it induces a chain isomorphism,

$$
C_{\bullet}(X ; N) \stackrel{\sim}{\longrightarrow} C_{\bullet}(M ; N),
$$

where $C_{\bullet}(M ; N)$ is the homology version of the complex from [1] and [3]. On the other hand,

$$
C_{\bullet}(X ; N) \simeq C_{\bullet}(\widetilde{X}) \otimes_{\mathbb{Z} \pi} N
$$

(isomorphism of chain complexes), for arbitrary $N$; see [26, Theorem VI.4.9]. At the same time,

$$
\operatorname{dim}_{\mathbb{C}}\left(C_{q}(\tilde{X}) \otimes_{\mathbb{Z} \pi} N\right)=\left(\operatorname{dim}_{\mathbb{C}} N\right) \cdot b_{q}(M(\mathcal{A})), \quad \forall q
$$

(from minimality), if $N$ is a finite-dimensional $\mathbb{C}$-vector space.

Eqs. (2.4), (2.7) and (2.6) together imply that the chain complex $C_{\bullet}(M ; N)$ computes the twisted homology of the complement, $H_{*}(M(\mathcal{A}) ; N)$; if $\operatorname{dim}_{\mathbb{C}} N=1,(2.8)$ implies that, furthermore, $\operatorname{dim}_{\mathbb{C}} C_{q}(M ; N)=b_{q}(M)$, for all $q$. In particular, we thus recover [3, Theorems 2.5 and 2.9], in homological form. 


\subsection{Principal ideal domain coefficients and minimal structures}

Let $X$ be a minimal $C W$-complex (connected, of finite type). Set $\pi:=\pi_{1}(X)$, and denote by $C \bullet(\widetilde{X})$ the $\pi$-equivariant chain complex from Section 2.1. By minimality,

$$
C_{q}(\tilde{X})=H_{q} X \otimes \mathbb{Z} \pi, \quad \text { for all } q .
$$

Set $R:=\mathbb{K} \mathbb{Z}$ (the group ring of $\mathbb{Z}$ over a commutative field $\mathbb{K}$ ). It is a principal ideal domain. We are going to use this fact to prove the following $R$-analog of Corollary 2.6, in the context of arbitrary minimal structures.

Let $\nu: \pi \rightarrow \mathbb{Z}$ be an arbitrary character. Extend it to a change of rings, $\nu: \mathbb{Z} \pi \rightarrow \mathbb{K} \mathbb{Z}$.

THEOREM 2.9. - Let $\varphi: X \rightarrow X^{\prime}$ be a homotopy equivalence, where both $X$ and $X^{\prime}$ are minimal $C W$-complexes. Use $\varphi_{\#}: \pi_{1}(X) \stackrel{\sim}{\rightarrow} \pi_{1}\left(X^{\prime}\right)$ to identify fundamental groups. Then the $\mathbb{K} \mathbb{Z}$-chain complexes $C_{\bullet}(\widetilde{X}) \otimes_{\mathbb{Z} \pi} \mathbb{K} \mathbb{Z}$ and $C_{\bullet}\left(\widetilde{X}^{\prime}\right) \otimes_{\mathbb{Z} \pi} \mathbb{K} \mathbb{Z}$ are isomorphic, for any change of rings homomorphism, $\nu: \mathbb{Z} \pi \rightarrow \mathbb{K} \mathbb{Z}$, as above.

The theorem follows from the lemma below, via the minimality property (2.9), and the basic homotopy invariance equation (2.4).

LEMma 2.10. - Let $C_{\bullet}=\left\{C_{q+1} \stackrel{d_{q+1}}{\longrightarrow} C_{q}\right\}_{q \geqslant 0}$ and $C_{\bullet}^{\prime}=\left\{C_{q+1}^{\prime} \stackrel{d_{q+1}^{\prime}}{\longrightarrow} C_{q}^{\prime}\right\}_{q \geqslant 0}$ be R-chain complexes, where $R$ is principal. Assume that $C_{q}$ and $C_{q}^{\prime}$ are finitely-generated free $R$-modules of the same rank, for all $q \geqslant 0$, and also that $H_{q}(C)$ and $H_{q}\left(C^{\prime}\right)$ are isomorphic $R$-modules, for $q \geqslant 0$. Then $C_{\bullet}$ and $C_{\bullet}^{\prime}$ are isomorphic $R$-chain complexes.

Proof. - We may easily infer from our assumptions that the submodules of $q$-cycles, $Z_{q}$ and $Z_{q}^{\prime}$, are $R$-free, of the same rank, $r_{q}$, for all $q$; likewise, the $q$-boundaries, $B_{q}$ and $B_{q}^{\prime}$, are both free of rank $s_{q}$, for all $q$.

Using suitable $R$-bases, the matrix of the inclusion, $B_{q} \hookrightarrow Z_{q}$, may be put in diagonal form, with nonzero entries, $\left\{a_{1}, \ldots, a_{s_{q}}\right\}$, on the diagonal, having the property that $a_{1}\left|a_{2}\right| \cdots \mid a_{s_{q}}$. Similarly, for $B_{q}^{\prime} \hookrightarrow Z_{q}^{\prime}$. Since $Z_{q} / B_{q} \simeq Z_{q}^{\prime} / B_{q}^{\prime}$, we infer that the elementary ideals generated by $s \times s$ minors must be equal, for $1 \leqslant s \leqslant s_{q}$; see [9, §20.2]. Hence, $a_{s}$ and $a_{s}^{\prime}$ differ by $R$-units, for all $s$. Therefore, we may find $R$-isomorphisms, $f_{q}: Z_{q} \stackrel{\sim}{\longrightarrow} Z_{q}^{\prime}$, inducing $R$-isomorphisms, $f_{q}: B_{q} \stackrel{\sim}{\longrightarrow} B_{q}^{\prime}$, for all $q \geqslant 0$.

At the same time, we may split $d_{q+1}: C_{q+1} \rightarrow B_{q}$, for $q \geqslant 0$, by choosing decompositions, $C_{q+1}=Z_{q+1} \oplus N_{q+1}$, such that $d_{q+1}: N_{q+1} \stackrel{\sim}{\longrightarrow} B_{q}$. Similarly, for $C_{\bullet}^{\prime}$. Extend $f_{q}: Z_{q} \stackrel{\sim}{\longrightarrow} Z_{q}^{\prime}$ to $f_{q}: C_{q} \stackrel{\sim}{\longrightarrow} C_{q}^{\prime}$, by setting $f_{q \mid N_{q}}=\left(d_{q}^{\prime}\right)^{-1} \circ\left(f_{q-1 \mid B_{q-1}}\right) \circ d_{q}: N_{q} \stackrel{\sim}{\longrightarrow} N_{q}^{\prime}$. By construction, the $R$-isomorphisms $\left\{f_{q}\right\}$ commute with differentials.

Theorem 2.9 may be applied to non-trivial higher homotopy groups of certain arrangements (which are very hard to compute, in general).

Let $\mathcal{A}$ be an essential projective arrangement in $\mathbb{P}^{r-1}$, with complement $M$ and fundamental group $\pi$. Assume that the cone, $\mathcal{A}^{\prime}$, is a hypersolvable central arrangement in $\mathbb{C}^{r}$ (see [15] for the definition and the basic properties of the hypersolvable class). Denote by $p:=p(M)$ the order of $\pi_{1}$-connectivity, introduced in [22]; it is a homotopy invariant of $M$, which turns out to be combinatorial, for the hypersolvable class, see [22, Corollary 4.10(1)]. Denote by $\widehat{\mathcal{A}}$ the fiber-type deformation of $\mathcal{A}$, constructed in [16].

If $p=r-1$, as in [8, Theorem 23], then [8, Theorem 18(ii)] applies to give the following $\mathbb{Z} \pi$-presentation for the first nonzero higher homotopy group of $M$ :

$$
\pi_{p}(M(\mathcal{A}))=\operatorname{coker}\left\{\partial_{p+2}: H_{p+2} M(\widehat{\mathcal{A}}) \otimes \mathbb{Z} \pi \rightarrow H_{p+1} M(\widehat{\mathcal{A}}) \otimes \mathbb{Z} \pi\right\},
$$


where $\partial_{p+2}$ is a boundary map from the $\pi$-equivariant chain complex (2.2), associated to a Morse-theoretic minimal structure on $M(\widehat{\mathcal{A}}) \cong K(\pi, 1)$.

One may associate to $\mathcal{A}^{\prime}$ a combinatorially determined collection of positive natural numbers, $\left\{1=d_{1}, d_{2}, \ldots, d_{\ell}\right\}$, called the exponents of $\mathcal{A}^{\prime}$, see [15]; they coincide with the exponents of the fiber-type central arrangement $\widehat{\mathcal{A}}^{\prime}$ defined in [10], see [16]. One knows [22, Lemma 5.3] that $\pi$ is an iterated semidirect product of free groups,

$$
\pi=\mathbb{F}_{d_{\ell}} \rtimes \cdots \rtimes \mathbb{F}_{d_{2}}
$$

with all monodromy actions trivial on homology.

The above structural property (2.11) has the following basic practical consequence, discovered by D. Cohen and A. Suciu in [6]: $K(\pi, 1)$ has a minimal structure for which all boundary maps of the associated $\pi$-equivariant chain complex,

$$
\partial_{q}^{\mathrm{Fox}}: H_{q} K(\pi, 1) \otimes \mathbb{Z} \pi \rightarrow H_{q-1} K(\pi, 1) \otimes \mathbb{Z} \pi,
$$

may be explicitly computed, by Fox differential calculus.

COROLlary 2.11. - Let $\mathcal{A}$ be an essential arrangement in $\mathbb{P}^{r-1}$, with hypersolvable cone, $\mathcal{A}^{\prime}$. Set $M:=M(\mathcal{A}), \pi:=\pi_{1}(M), p:=p(M)$. Assume that $p=r-1$. Let $\nu: \mathbb{Z} \pi \rightarrow \mathbb{K} \mathbb{Z}$ be the change of rings associated to an arbitrary character, $\nu: \pi \rightarrow \mathbb{Z}$ (where $\mathbb{K}$ is a commutative field). Then:

$$
\pi_{p}(M(\mathcal{A})) \otimes_{\mathbb{Z} \pi} \mathbb{K} \mathbb{Z}=\operatorname{coker}\left\{\partial_{p+2}^{\mathrm{Fox}} \otimes_{\mathbb{Z} \pi} \mathbb{K} \mathbb{Z}:(\mathbb{K} \mathbb{Z})^{b_{p+2}(\pi)} \rightarrow(\mathbb{K} \mathbb{Z})^{b_{p+1}(\pi)}\right\}
$$

as $\mathbb{K} \mathbb{Z}$-modules, where the Betti numbers of $\pi$ are determined by the exponents of $\mathcal{A}^{\prime}$, and $\partial_{\bullet}^{\text {Fox }}$ is explicitly computed from (2.11).

Proof. - The Poincaré polynomial of $K(\pi, 1) \cong M(\widehat{\mathcal{A}})$ is $P(T)=\prod_{i=2}^{\ell}\left(1+d_{i} T\right)$; see [10]. From (2.10), we infer that $\pi_{p}(M) \otimes_{\mathbb{Z} \pi} \mathbb{K} \mathbb{Z}=\operatorname{coker}\left\{\partial_{p+2} \otimes_{\mathbb{Z} \pi} \mathbb{K} \mathbb{Z}\right\}$. By Theorem 2.9, we may replace $\partial_{p+2}$ by $\partial_{p+2}^{\text {Fox }}$, to arrive at (2.12), as asserted.

\section{A Milnor fiber obstruction to relative minimality}

\subsection{Absolute minimality and $H_{1}$-bases}

We come back to Definition 2.3 from the preceding section. We will add more information, related to distinguished $H_{1}$-bases. This will be needed for certain twisted homology computations (such as those related to the homology of Milnor fibers).

Let $\left\{H_{0}, H_{1}, \ldots, H_{n}\right\}$ be the hyperplanes of $\mathcal{A}$, in $\mathbb{P}(U)$. The meridians associated to the hyperplanes, $\left\{\mu_{i} \in \pi_{1}(M)\right\}_{0 \leqslant i \leqslant n}$, give a collection of well-defined elements of $H_{1} M$. Considering $H_{0}$ as a distinguished hyperplane, we obtain in this way a distinguished $\mathbb{Z}$-basis of $H_{1} M$,

$$
\left\{\left[\mu_{i}\right]\right\}_{1 \leqslant i \leqslant n}
$$

Let now $\left\{c_{i}\right\}_{1 \leqslant i \leqslant n}$ be the 1 -cells of a minimal $C W$-structure on $M, X$. They provide a distinguished $\mathbb{Z}$-basis of $H_{1} X$,

$$
\left\{\left[c_{i}\right]\right\}_{1 \leqslant i \leqslant n}
$$


Definition 3.2. - Let $X$ be a minimal complex. A homotopy equivalence, $\varphi: X \rightarrow M$, respects $H_{1}$-markings, if it takes the basis (3.2) to the basis (3.1).

Every arrangement complement, $M$, has such a marked minimal structure. Indeed, take an $\mathcal{A}$-generic flag $\mathcal{F}$, as in Definition 2.2, and consider an $\mathcal{F}$-compatible minimal structure, as in Definition 2.3. It is constructed inductively, see [8, Section 4]. At the first nontrivial step, $X^{(1)}$ is a wedge of $n$ circles, and $F_{1} M$ is $\mathbb{P}^{1} \backslash\{n+1$ points $\}$. Obviously, we may start with a homotopy equivalence, $\varphi_{1}$, which preserves the canonical $H_{1}$-bases, and then proceed by induction.

\subsection{The relative minimality problem}

Assume now that $\mathcal{A}$ is an $\mathcal{L}_{k}(\widehat{\mathcal{A}})$-generic section of $\widehat{\mathcal{A}}$ (in the sense from [8, (1)]), with $k \geqslant 1$, where $\widehat{\mathcal{A}}$ is an essential arrangement of $n+1$ hyperplanes in $\mathbb{P}(V)$. Our basic idea in [8] was to extract from $\widehat{\mathcal{A}}$ homotopy information on $M(\mathcal{A})$. The key tool is provided by [8, Proposition 14], which says that one may replace, up to homotopy, the inclusion, $j: M(\mathcal{A}) \hookrightarrow M(\widehat{\mathcal{A}})$, by a cellular map between minimal complexes, $f: X \rightarrow Y$, with the property that

$$
f_{\mid X^{(k)}}=\mathrm{id}
$$

The relative minimality problem we have in mind is the following. Start with a marked minimal structure on $M(\widehat{\mathcal{A}}), Y$, as in Definition 3.2. Let $\mathcal{A}$ be an essential $\mathcal{L}_{k}$-generic section of $\widehat{\mathcal{A}}$, with $k \geqslant 1$. Can one replace $j$ by $f$, as in (3.3) above, in such a way that moreover

$$
f_{\mid X^{(k+1)}}=\text { subcomplex inclusion? }
$$

When $\mathcal{A}$ has hypersolvable cone and $p:=p(M(\mathcal{A}))=\operatorname{rank}\left(\mathcal{A}^{\prime}\right)-1$, as in Corollary 2.11, this can be done: actually one may take $X=Y^{(p)}$, and $f=$ inclusion; see [8, Theorems 18 and 23]. In general, the (easily checked) fact that $H_{*} j$ is a split injection, together with heuristic morsification arguments, seem to indicate that the answer to question (3.4) should be yes.

Surprisingly enough, the answer turns out to be no, in general, and the homology of the Milnor fiber of $\mathcal{A}$ comes into play, at this point.

\subsection{Twisted homology and Betti numbers of Milnor fibers}

Let $q:=\prod_{i=0}^{n} \alpha_{i}$ be a defining equation of the central arrangement in $U$ associated to $\mathcal{A}$. Let $F:=q^{-1}(1)$ be the Milnor fiber of $\mathcal{A}$. We are going to recall from [4, Corollary 1.5] the twisted homology decomposition of $H_{*}(F ; \mathbb{C})$.

Set $u:=\exp \left(\frac{2 \pi \sqrt{-1}}{n+1}\right)$. For $0 \leqslant t \leqslant n$, denote by $L_{t}$ the rank one $\mathbb{C}$-local system on $M:=M(\mathcal{A})$ (alias, the abelian representation, $L_{t}: H_{1} M \rightarrow \mathbb{C}^{*}$, of $\left.\pi_{1}(M)\right)$ given by

$$
L_{t}\left(\left[\mu_{i}\right]\right)=u^{t}, \quad \text { for } 1 \leqslant i \leqslant n
$$

(where $\left\{\left[\mu_{i}\right]\right\}$ is the $\mathbb{Z}$-basis (3.1)). Set

$$
b_{s}^{t}(F):=\operatorname{dim}_{\mathbb{C}} H_{s}\left(M ; L_{t}\right) .
$$

Then:

$$
b_{s}(F)=\sum_{t=0}^{n} b_{s}^{t}(F), \quad \text { for all } s
$$




\subsection{The Milnor fiber obstruction}

Let $\mathcal{A}$ be an arbitrary essential arrangement in $\mathbb{P}(U)$, with defining equation $q$ and Milnor fiber $F$, as in Section 3.4. The linear forms $\left\{\alpha_{i}\right\}_{0 \leqslant i \leqslant n}$ define a linear embedding

$$
j: U \hookrightarrow V:=\mathbb{C}^{n+1},
$$

which enables us to view $\mathcal{A}$ as an $\mathcal{L}_{1}$-generic section of the Boolean arrangement $\widehat{\mathcal{A}}$ (with defining equation $\left.\widehat{q}:=\prod_{i=0}^{n} z_{i}\right)$.

Set $Y:=\left(S^{1}\right)^{\times n}$, endowed with the canonical minimal structure of the $n$-torus. Plainly, there is a marked homotopy equivalence, $\varphi_{Y}: Y \rightarrow M(\widehat{\mathcal{A}})$. Assume that $j: M(\mathcal{A}) \hookrightarrow M(\widehat{\mathcal{A}})$ has the homotopy type of a cellular map between minimal complexes, $f: X \rightarrow Y$, with the property that $f_{\mid X^{(1)}}=\mathrm{id}$, as in (3.3), and $f_{\mid X^{(2)}}=$ inclusion, as in (3.4). (Note that $\varphi_{X}: X \rightarrow M(\mathcal{A})$ will also be a marked homotopy equivalence, since, obviously, $j$ and $f$ respect $H_{1}$-markings.)

THEOREM 3.6. - Let $\mathcal{A}$ be an essential arrangement of $n+1$ hyperplanes, with Milnor fiber $F$. If the relative minimality problem (3.4), where $k=1$, has a positive answer, for $\mathcal{A}$ and the Boolean arrangement $\widehat{\mathcal{A}}$, then $n$ divides $b_{1}(F)$.

Proof. - Since $b_{1}^{0}(F)=b_{1}(M(\mathcal{A}))=n$, it will be enough to show that $b_{1}^{t}(F)$ is independent of $t$, for $1 \leqslant t \leqslant n$; see (3.7). As $\varphi_{X}$ is a marked homotopy equivalence, the twisted Betti numbers from (3.6) may be computed on $X$, using the cellular $H_{1}$-basis (3.2) in (3.5), via the basic specialization formula (2.4).

Our hypothesis (3.3) on $f_{\mid X^{(1)}}$ readily implies that $f_{\#}: \pi:=\pi_{1}(X) \rightarrow \pi_{1}(Y)=\mathbb{Z}^{n}$ is the

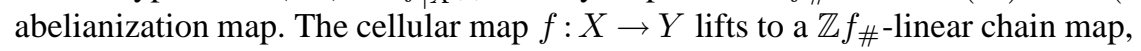

$$
\left\{\widetilde{f}_{s}: H_{s} X \otimes \mathbb{Z} \pi \rightarrow H_{s} Y \otimes \mathbb{Z} \mathbb{Z}^{n}\right\}_{s \geqslant 0}
$$

between the equivariant chain complexes of the universal covers,

$$
C \bullet(\tilde{X}):=\left\{H_{s+1} X \otimes \mathbb{Z} \pi \stackrel{d_{s+1}}{\longrightarrow} H_{s} X \otimes \mathbb{Z} \pi\right\}_{s \geqslant 0},
$$

and

$$
C \cdot(\tilde{Y}):=\left\{H_{s+1} Y \otimes \mathbb{Z}^{n} \stackrel{\partial_{s+1}}{\longrightarrow} H_{s} Y \otimes \mathbb{Z}^{n}\right\}_{s \geqslant 0} .
$$

Our main hypothesis (3.4) implies that

$$
\widetilde{f}_{s}=H_{s} f \otimes \mathbb{Z} f_{\#}, \quad \text { for } s \leqslant 2,
$$

with $H_{\leqslant 2} f$ monic.

For $s \leqslant 1$, we may thus tensor the commutative squares

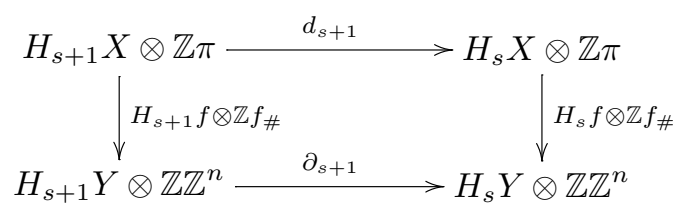


(see (3.9)) with $\mathbb{Z} \mathbb{Z}^{n}$ over $\mathbb{Z} \pi$, via $\mathbb{Z} f_{\#}$, to get commuting squares

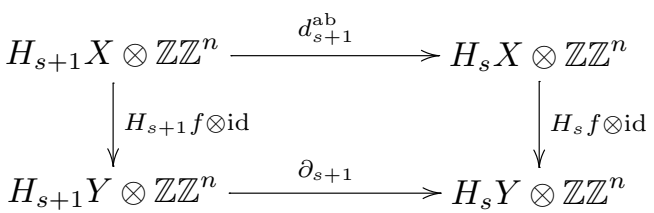

For any $1 \leqslant t \leqslant n$, we may further specialize to $\mathbb{C}$, via the representation (3.5). In this way, we get commutative squares,

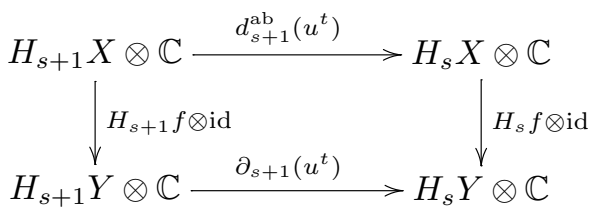

(for $s \leqslant 1$ ), where the vertical maps are injective, and independent of $t$. Note that the upper chain complex from (3.10) computes $b_{1}^{t}(F)$; see (3.6) and (2.4).

At the same time, eye-inspection of the well-known explicit formula for $\left\{\partial_{s+1}\right\}_{s}$ (see e.g. [8, (10)]) reveals that $\partial_{s+1}\left(u^{t}\right)=\left(u^{-t}-1\right) \cdot \partial_{s+1}^{\prime}$, where the differential $\partial_{s+1}^{\prime}$ is independent of $t$. By (3.10) above, $b_{1}^{t}(F)$ is therefore independent of $t$, for $0<t \leqslant n$.

EXAMPLE 3.7. - In Example 5.1 from [4], $n=5, b_{1}^{t}=0$, for $t=1,3,5$, and $b_{1}^{t}=1$, for $t=2,4$; hence, $b_{1}(F)=7$. In Example 5.4 from [4], $n=8, b_{1}^{t}=0$, for $t=1,2,4,5,7,8$, and $b_{1}^{t}=1$, for $t=3,6$; hence, $b_{1}(F)=10$. By Theorem 3.6, the relative minimality problem (3.4) has a negative answer, in both cases.

\section{Twisted homology with resonant coefficients}

Let $\mathcal{A}$ be an essential, proper, $\mathcal{L}_{k}(\widehat{\mathcal{A}})$-generic section, with $k \geqslant 2$, of an essential aspherical arrangement, $\widehat{\mathcal{A}}$. (When we say 'proper', we want to exclude the trivial case, $\widehat{\mathcal{A}}=\mathcal{A}$.) Set $M:=M(\mathcal{A}), \pi:=\pi_{1}(M)$, and $p:=p(M)$.

We know that $j: M(\mathcal{A}) \hookrightarrow M(\widehat{\mathcal{A}})$ has the homotopy type of a cellular map between minimal complexes, $f: X \rightarrow Y$, with the property that

$$
f_{\mid X^{(p)}}=\mathrm{id},
$$

where $2 \leqslant k \leqslant p<\infty$. In particular, $Y$ is a $K(\pi, 1)$. See the discussion preceding Theorem $16[8]$.

Our goal in this section is to use (4.1) above, to perform various twisted homology computations on $M$, in terms of $\pi_{1}(M)$. We will not impose any kind of 'nonresonance' conditions on the coefficients.

\subsection{Computations in the $\mathcal{L}$-generic range}

Here the coefficients will be quite general, but our method will give results only in the $\mathcal{L}$-generic range, that is, up to $H_{p-1}(M ; L)$. 
THEOREM 4.2. - Let $\mathcal{A}$ be an essential, proper, $\mathcal{L}_{k}(\widehat{\mathcal{A}})$-generic section, with $k \geqslant 2$, of an essential aspherical arrangement, $\widehat{\mathcal{A}}$. Set $M:=M(\mathcal{A}), \pi:=\pi_{1}(M)$, and $p:=p(M)$.

(1) Let $L$ be an arbitrary local system on $M$. Then:

$$
H_{q}(M ; L)=\operatorname{Tor}_{q}^{\mathbb{Z} \pi}(\mathbb{Z}, L), \quad \text { for } q<p .
$$

(2) If $\widehat{\mathcal{A}}$ is fiber-type, then the $\mathrm{Tor}^{\mathbb{Z} \pi}$-groups from part (1) may be explicitly computed using the Fox $\mathbb{Z} \pi$-resolution of $\mathbb{Z}$ from $[6]$.

Proof. - Part (1). By (2.4) and basic homotopy formula (4.1),

$$
H_{<p}(M ; L)=H_{<p}\left(C \bullet(\widetilde{Y}) \otimes_{\mathbb{Z} \pi} L\right) .
$$

The identification of $H_{<p}\left(C \bullet(\widetilde{Y}) \otimes_{\mathbb{Z} \pi} L\right)$ with $\operatorname{Tor}_{<p}^{\mathbb{Z} \pi}(\mathbb{Z}, L)$ comes now from the fact that the $\pi$-equivariant chain complex $C_{\bullet}(\widetilde{Y})$ is a free $\mathbb{Z} \pi$-resolution of $\mathbb{Z}$, since $Y$ is a $K(\pi, 1)$.

Part (2). If $\widehat{\mathcal{A}}$ is fiber-type, then $\pi=\pi_{1}(M(\widehat{\mathcal{A}}))$ is an iterated semidirect product of free groups, with trivial monodromy actions on homology, as in (2.11). Therefore, the Fox calculus free $\mathbb{Z} \pi$-resolution of $\mathbb{Z}$ from [6] may be used as well to compute the $\operatorname{Tor}^{\mathbb{Z} \pi}$-groups from part (1).

As an illustration of Theorem 4.2, we may offer the following simple, very explicit, class of examples. Let $\mathcal{A}$ be an essential projective arrangement of $n+1$ hyperplanes, with associated central arrangement, $\mathcal{A}^{\prime}$. Given a subarrangement, $\mathcal{B}^{\prime} \subset \mathcal{A}^{\prime}$, denote by $\left|\mathcal{B}^{\prime}\right|$ the number of hyperplanes of $\mathcal{B}^{\prime}$.

Define $c(\mathcal{A}):=\infty$, if $\mathcal{A}^{\prime}$ is independent. Otherwise, set

$$
c(\mathcal{A}):=\min \left\{\left|\mathcal{B}^{\prime}\right| \mid \mathcal{B}^{\prime} \subset \mathcal{A}^{\prime} \text { is dependent }\right\} .
$$

Obviously, $c(\mathcal{A}) \geqslant 3$.

Corollary 4.3. - Let $\mathcal{A}$ be an essential arrangement of $n+1$ hyperplanes in $\mathbb{P}(U)$. Set $M:=M(\mathcal{A}), \pi:=\pi_{1}(M), p:=p(M)$, and $c:=c(\mathcal{A})$.

(1) If $c<\infty$, then $\mathcal{A}$ is a proper $\mathcal{L}_{c-2}$-generic section of an essential Boolean arrangement of $n+1$ hyperplanes.

(2) If $c>3$, then $\pi=\mathbb{Z}^{n}$ and $p=c-2$.

(3) Assume $3<c<\infty$. Let $L$ be a left $\mathbb{Z} \mathbb{Z}^{n}$-module. Define a chain complex,

$$
C \bullet\left(\mathbb{Z}^{n} ; L\right):=\left\{\bigwedge^{s}\left(x_{1}, \ldots, x_{n}\right) \otimes_{\mathbb{Z}} L \stackrel{\partial_{s}}{\longrightarrow} \bigwedge^{s-1}\left(x_{1}, \ldots, x_{n}\right) \otimes_{\mathbb{Z}} L\right\}_{s \geqslant 1},
$$

by setting

$$
\partial_{s}\left(x_{i_{1}} \wedge \cdots \wedge x_{i_{s}} \otimes v\right):=\sum_{r=1}^{s}(-1)^{r-1} x_{i_{1}} \wedge \cdots \widehat{x_{i_{r}}} \cdots \wedge x_{i_{s}} \otimes\left(x_{i_{r}}^{-1}-1\right) v,
$$

where $\left\{x_{1}, \ldots, x_{n}\right\}$ denotes the standard basis of $\mathbb{Z}^{n}$. Then:

$$
H_{q}(M ; L)=H_{q}\left(C \bullet\left(\mathbb{Z}^{n} ; L\right)\right), \quad \text { for } q<c-2 .
$$

In particular, the $L$-twisted homology of $M(\mathcal{A})$ in the $\mathcal{L}$-generic range depends only on $L$, and the combinatorics of $\mathcal{A}$. 
Proof. - Part (1). Eq. (3.8) from Section 3.5 shows that $\mathcal{A}$ is an $\mathcal{L}_{1}$-generic section (proper, since $c<\infty$ ) of the required Boolean arrangement, $\widehat{\mathcal{A}}$. This section may be easily seen to be actually $\mathcal{L}_{c-2}$-generic, by resorting to the definitions: see $[8,(1)]$ for $\mathcal{L}_{k}$-genericity, and (4.2) above for $c(\mathcal{A})$.

Part (2). If $c=\infty$, then plainly $\mathcal{A}$ itself is Boolean, $\pi=\mathbb{Z}^{n}$ and $p=\infty$. Assume then that $3<c<\infty$. By [8, Proposition 14], $\pi=\mathbb{Z}^{n}$ and $M(\widehat{\mathcal{A}})$ is a $K(\pi, 1)$. Moreover, $k(\widehat{\mathcal{A}}, U)=$ $p(\widehat{\mathcal{A}}, U)$, according to [8, Proposition 15]. One may check the equality $k(\widehat{\mathcal{A}}, U)=c-2$, directly from the definitions $([8,(3)]$ and (4.2) respectively). Likewise, the equality $p(\widehat{\mathcal{A}}, U)=p(M(\mathcal{A}))$ follows from the definitions ([8, (4)] and [22, p. 73] respectively). Finally, $p=c-2$, as asserted.

Part (3). Parts (1)-(2) enable us to compute $H_{<c-2}(M ; L)$ as in Theorem 4.2(1). The equality $H_{q}\left(C \bullet\left(\mathbb{Z}^{n} ; L\right)\right)=\operatorname{Tor}_{q}^{\mathbb{Z} \mathbb{Z}^{n}}(\mathbb{Z}, L)$ follows at once, by using the standard $\mathbb{Z} \mathbb{Z}^{n}$-resolution of $\mathbb{Z}[8$, (10)] to compute $\operatorname{Tor}^{\mathbb{Z} \mathbb{Z}^{n}}$-groups.

\subsection{A fiber-type general position framework for complete computations}

Now we are going to focus on the fiber-type general position class from Corollary 2.11. By definition, an essential arrangement $\mathcal{A}$ belongs to this class if it has hypersolvable cone, $\mathcal{A}^{\prime}$, and $p:=p(M(\mathcal{A}))=r-1$, where $r:=\operatorname{rank}\left(\mathcal{A}^{\prime}\right)$. We know that $p \geqslant 2$, and that this class coincides with the proper $\mathcal{L}_{r-1}$-generic sections, $r \geqslant 3$, of essential fiber-type arrangements, $\widehat{\mathcal{A}}$; see the discussion preceding Theorem 23 [8].

When $\widehat{\mathcal{A}}$ is Boolean, the above definition corresponds to the general position arrangements, intensively studied since Hattori's pioneering work [13]; see [8, Remark 19]. This explains both our terminology, and our interest in the fiber-type general position class.

Let $\mathbb{K}$ be a commutative field. We will treat two types of local coefficients on $M:=M(\mathcal{A})$. The first type consists of $P I D$ coefficients, $R:=\mathbb{K} \mathbb{Z}$, coming from characters, $\nu: \pi \rightarrow \mathbb{Z}$, as in Section 2.8. The second type consists of $\pi$-modules $N:=\mathbb{K}^{d}$, coming from finite-dimensional $\mathbb{K}$-representations, $\rho: \pi \rightarrow G L(d ; \mathbb{K})$. In both cases, our choice was guided by the desire to have an Euler characteristic argument at hand, to obtain complete computations.

THEOREM 4.5. - Let $\mathcal{A}$ be an essential arrangement in $\mathbb{P}^{r-1}$, with $r \geqslant 3$. Set $M:=M(\mathcal{A})$ and $\pi:=\pi_{1}(M)$. Assume that $\mathcal{A}$ is a proper, $\mathcal{L}_{r-1}(\widehat{\mathcal{A}})$-generic section, of an essential aspherical arrangement, $\widehat{\mathcal{A}}$. Let $\mathbb{K}$ be a commutative field.

(1) Set $R:=\mathbb{K} \mathbb{Z}$, endowed with the left $\mathbb{Z} \pi$-module structure coming from a character, $\nu: \pi \rightarrow \mathbb{Z}$. Then:

$$
H_{q}(M(\mathcal{A}) ; R)= \begin{cases}\operatorname{Tor}_{q}^{\mathbb{Z} \pi}(\mathbb{Z}, R), & \text { for } q<r-1 \\ R \text {-free, of rank }=(-1)^{r-1}\left[\chi(M(\mathcal{A}))-\kappa_{r-1}\right], & \text { for } q=r-1 \\ 0, & \text { for } q>r-1\end{cases}
$$

where $\kappa_{r-1}=\sum_{q=0}^{r-2}(-1)^{q} \operatorname{rank}_{R} \operatorname{Tor}_{q}^{\mathbb{Z} \pi}(\mathbb{Z}, R)$.

(2) Set $N:=\mathbb{K}^{d}$, endowed with the left $\mathbb{Z} \pi$-module structure coming from a representation, $\rho: \pi \rightarrow G L(d ; \mathbb{K})$. Then:

$$
H_{q}(M(\mathcal{A}) ; N)= \begin{cases}\operatorname{Tor}_{q}^{\mathbb{Z} \pi}(\mathbb{Z}, N), & \text { for } q<r-1 \\ \text { of } \mathbb{K}-\operatorname{dim}=(-1)^{r-1}\left[d \cdot \chi(M(\mathcal{A}))-\kappa_{r-1}\right], & \text { for } q=r-1 \\ 0, & \text { for } q>r-1\end{cases}
$$

where $\kappa_{r-1}=\sum_{q=0}^{r-2}(-1)^{q} \operatorname{dim}_{\mathbb{K}} \operatorname{Tor}_{q}^{\mathbb{Z} \pi}(\mathbb{Z}, N)$. 
(3) If $\widehat{\mathcal{A}}$ is fiber-type (e.g., if $\mathcal{A}$ has hypersolvable cone, and $p(M(\mathcal{A}))=r-1)$, then all Tor $^{\mathbb{Z} \pi}$ computations from parts (1) and (2) may be done explicitly, with the aid of the Fox $\mathbb{Z} \pi$-resolution of $\mathbb{Z}$ from [6].

Proof. - Set $p:=p(M)$. We know that $p=r-1$, by [8, Theorem 18(i)]. For $q<r-1$, our assertions from parts (1)-(2) follow then directly from Theorem 4.2(1). If $q>r-1$, then plainly $H_{q}(M ; L)=0$ ( $L$ arbitrary), for dimensional reasons. It remains to settle the case $q=r-1$, for $R$ and for $N$.

Denote by $X$ (respectively $Y$ ) the minimal $C W$-structure on $M(\mathcal{A})$ (respectively on $M(\widehat{\mathcal{A}})$ ). We infer from (4.1) that $X=Y^{(r-1)}$, where $Y$ is a $K(\pi, 1)$. Therefore (see (2.4)), $H_{r-1}(M ; L)=\operatorname{ker}\left\{\partial_{r-1} \otimes_{\mathbb{Z} \pi} L: H_{r-1} Y \otimes_{\mathbb{Z}} L \rightarrow H_{r-2} Y \otimes_{\mathbb{Z}} L\right\}$, for arbitrary $L$, where $\left\{\partial_{r-1}\right\}_{r \geqslant 2}$ denotes the differential of the $\pi$-equivariant chain complex $C_{\bullet}(\tilde{Y})$.

In part (1), we may use an Euler characteristic argument for the finite, $R$-free, $R$-chain complex $C \bullet\left(\widetilde{Y}^{(r-1)}\right) \otimes_{\mathbb{Z} \pi} R$, to infer that $H_{r-1}(M ; R)$ is $R$-free and

$$
\chi(M)=(-1)^{r-1} \operatorname{rank}_{R} H_{r-1}(M ; R)+\kappa_{r-1},
$$

as asserted.

In part (2), we may apply the same argument to the finite $\mathbb{K}$-chain complex $C \bullet\left(\tilde{Y}^{(r-1)}\right) \otimes_{\mathbb{Z} \pi} N$, to get

$$
d \cdot \chi(M)=(-1)^{r-1} \operatorname{dim}_{\mathbb{K}} H_{r-1}(M ; N)+\kappa_{r-1},
$$

which verifies our claimed formula.

As for part (3), we may use the same argument as in the proof of Theorem 4.2(2).

\section{Character-abelianizations of higher homotopy groups}

\subsection{Nonresonant abelianization and combinatorics}

We continue to study the homotopy properties of the complement, for arrangements belonging to the fiber-type general position class from Corollary 2.11. Within this class, we have explained, in Section 2.8, how to construct an explicit presentation matrix for $\pi_{p}(M) \otimes_{\mathbb{Z} \pi} \mathbb{K} \mathbb{Z}$, for an arbitrary character, $\nu: \pi \rightarrow \mathbb{Z}$.

A priori, only the size of the presentation matrix $\partial_{p+2}^{\text {Fox }} \otimes_{\mathbb{Z} \pi} \mathbb{K} \mathbb{Z}$ from Corollary 2.11 is combinatorially determined. In this subsection, we aim at enlarging the dictionary 'topology $\leftrightarrow$ combinatorics', along the lines from [8]; see especially Theorem 23 therefrom.

More precisely, we would like to identify more numerical invariants of $\pi_{p}(M(\mathcal{A}))$ which are determined by the combinatorics of $\mathcal{A}$. A natural candidate is provided by $\operatorname{rank}_{R} \pi_{p}(M) \otimes_{\nu} R$, where $R:=\mathbb{K} \mathbb{Z}$. To derive a combinatorial formula for the aforementioned $R$-rank, we are led to impose a certain 'nonresonance' condition on the local system $\nu$.

This condition in turn is inspired from the powerful vanishing result, for $\mathbb{C}$-local systems of finite rank, proved in [2]. To make the appropriate formal definition, we recall that there is a combinatorially defined subset of edges, $\mathcal{D}(\mathcal{A}) \subset \mathcal{L}(\mathcal{A})$, called dense edges, for any arrangement $\mathcal{A}$; see [25].

DEFINITION 5.2. - Let $\mathcal{A}=\left\{H_{0}, H_{1}, \ldots, H_{n}\right\}$ be an essential arrangement, with fundamental group $\pi$. A character, $\nu: \pi \rightarrow \mathbb{Z}$ (that is, a collection $\left\{\gamma_{i}:=\nu\left(\mu_{i}\right) \in \mathbb{Z}\right\}_{0 \leqslant i \leqslant n}$, such that 
$\left.\sum_{i=0}^{n} \gamma_{i}=0\right)$ is called $\mathcal{A}$-nonresonant if

$$
\sum_{H_{i} \supset S} \gamma_{i} \neq 0, \quad \forall S \in \mathcal{D}(\mathcal{A}) \text { such that } S \subset H_{0}
$$

Given $\mathcal{A}$, it is straightforward to check that $\mathcal{A}$-nonresonant characters always exist.

THEOREM 5.3. - Let $\mathcal{A}$ and $\widehat{\mathcal{A}}$ be essential arrangements, in $\mathbb{P}^{r-1}$ and $\mathbb{P}^{m-1}$ respectively. Assume that $\widehat{\mathcal{A}}$ is aspherical, and $\mathcal{A}$ is a proper, $\mathcal{L}_{r-1}(\widehat{\mathcal{A}})$-generic section of $\widehat{\mathcal{A}}$, with $r \geqslant 3$. Set $M:=M(\mathcal{A})$ and $\pi:=\pi_{1}(M)$.

(1) $\pi_{r-1}(M(\mathcal{A}))$ is the first higher non-vanishing homotopy group of $M$.

(2) Let $\nu: \pi \rightarrow \mathbb{Z}$ be $\widehat{\mathcal{A}}$-nonresonant (in the sense of Definition 5.2 above). Set $R:=\mathbb{C} \mathbb{Z}$. Then:

$$
\operatorname{rank}_{R} \pi_{r-1}(M(\mathcal{A})) \otimes_{\nu} R= \begin{cases}(-1)^{r-1} \chi(M(\mathcal{A})), & \text { if } r+1<m ; \\ b_{r}(\pi), & \text { if } r+1=m .\end{cases}
$$

(3) If $\mathcal{A}$ has hypersolvable cone, $p(M(\mathcal{A}))=r-1$, and $\widehat{\mathcal{A}}$ is the fiber-type deformation of $\mathcal{A}$ from [16], then $b_{r}(\pi)$ from the second case in part (2) equals $\prod_{j=1}^{\ell} d_{j}$, where $\left\{d_{j}\right\}$ are the exponents of the cone of $\mathcal{A}$. In particular, $\operatorname{rank}_{R} \pi_{r-1}(M(\mathcal{A})) \otimes_{\nu} R$ is combinatorially determined, if $\nu$ is $\widehat{\mathcal{A}}$-nonresonant.

Proof. - Part (1) follows directly from [8, Theorem 18]. Let $Y$ be the minimal $C W$-structure on $M(\widehat{\mathcal{A}})$ from [8, Theorem 18(ii)], with associated $\pi$-equivariant chain complex,

$$
C_{\bullet}(\tilde{Y}):=\left\{\partial_{q}: H_{q} Y \otimes \mathbb{Z} \pi \rightarrow H_{q-1} Y \otimes \mathbb{Z} \pi\right\}_{q \geqslant 1} .
$$

We infer, from the $\mathbb{Z} \pi$-resolution $[8,(11)]$ of $\pi_{r-1}(M)$, that

$$
\pi_{r-1}(M) \otimes_{\nu} R=\operatorname{coker}\left\{H_{r+1} Y \otimes_{\mathbb{Z}} R \stackrel{\partial_{r+1} \otimes_{\nu} R}{\longrightarrow} H_{r} Y \otimes_{\mathbb{Z}} R\right\},
$$

for arbitrary $\nu$.

Part (2). If $r+1=m$, then $\pi_{r-1}(M) \otimes_{\nu} R=H_{r} Y \otimes R$ is $R$-free, with $R$-rank equal to $b_{r}(\pi)$, as asserted, for arbitrary $\nu$ (since $Y$ is a $K(\pi, 1)$ ). Assume then that $r+1<m$.

In this case, $\widehat{\mathcal{A}}$-nonresonance comes into play, in the following way. As a preliminary remark, note that the chain complex, $C_{\bullet}(\widetilde{Y}) \otimes_{\mathbb{Z} \pi} \mathbb{C}_{t}:=\left\{\partial_{q+1} \otimes_{\nu} R(t): H_{q+1} Y \otimes \mathbb{C} \rightarrow H_{q} Y \otimes \mathbb{C}\right\}_{q \geqslant 0}$, obtained by further specializing $1 \in \mathbb{Z}$ to $t \in \mathbb{C}^{*}$, computes the homology of $M(\widehat{\mathcal{A}})$ with coefficients in the appropriately defined rank one $\mathbb{C}$-local system, $\mathbb{C}_{t}$; see (2.4).

Therefore, Theorem 1 and Lemma 2 from [2] together imply that

$$
H_{\neq m-1}\left(C_{\bullet}(\tilde{Y}) \otimes_{\mathbb{Z} \pi} \mathbb{C}_{t}\right)=0,
$$

as soon as

$$
{ }_{t}^{\sum_{H_{i} \supset S} \gamma_{i}} \neq 1, \quad \forall S \in \mathcal{D}(\widehat{\mathcal{A}}) \text { such that } S \subset H_{0} .
$$

Comparing (5.4) with (5.1), we infer from $\widehat{\mathcal{A}}$-nonresonance that the vanishing property (5.3) holds, for generic $t$.

Recall now from (4.1) that $Y^{(r-1)}$ is a minimal $C W$-structure for $M(\mathcal{A})$. An Euler characteristic argument, applied to the $\mathbb{C}$-chain complex $C_{\bullet}\left(\widetilde{Y}^{(r-1)}\right) \otimes_{\mathbb{Z} \pi} \mathbb{C}_{t}$, for generic $t$, provides the equality 


$$
(-1)^{r-1} \chi(M(\mathcal{A}))=\operatorname{dim}_{\mathbb{C}} \operatorname{ker}\left\{\partial_{r-1} \otimes_{\nu} R(t)\right\} .
$$

Since $r<m-1$, (5.3) also implies that

$$
\operatorname{ker}\left\{\partial_{r-1} \otimes_{\nu} R(t)\right\}=\operatorname{coker}\left\{\partial_{r+1} \otimes_{\nu} R(t)\right\},
$$

for generic $t$. Using Eqs. (5.5), (5.6) and (5.2), we arrive at

$$
\operatorname{dim}_{\mathbb{C}}\left(\pi_{r-1}(M) \otimes_{\nu} R\right) \otimes_{R} \mathbb{C}_{t}=(-1)^{r-1} \chi(M(\mathcal{A}))
$$

for generic $t$.

At the same time, it is well-known that $\operatorname{rank}_{R} N=\operatorname{dim}_{\mathbb{C}}\left(N \otimes_{R} \mathbb{C}_{t}\right)$, generically, for a finite $R$-module $N$. With this remark, Eq. (5.7) finishes the proof of part (2).

Part (3). In the hypersolvable case, it is enough to recall from [10] that the Poincaré polynomial of $M(\widehat{\mathcal{A}}) \cong K(\pi, 1)$ is $\prod_{j=2}^{\ell}\left(1+d_{j} T\right)$, where $\ell=m$, and $d_{1}=1$; see also our discussion preceding Corollary 2.11 from Section 2.8.

REMARK 5.4. - Let $\mathcal{A}$ be an arbitrary essential arrangement in $\mathbb{P}^{r-1}$, with fundamental group $\pi$. Set $R:=\mathbb{C} \mathbb{Z}$. Let $\nu: \pi \rightarrow \mathbb{Z}$ be $\mathcal{A}$-nonresonant. Then it is not difficult to use the same kind of arguments as in the proof of Theorem 4.2 from [7], to obtain that

$$
H_{q}(M(\mathcal{A}) ; R)= \begin{cases}R \text {-torsion, } & \text { for } q<r-1 \\ R \text {-free, of } \operatorname{rank}=(-1)^{r-1} \chi(M(\mathcal{A})), & \text { for } q=r-1 \\ 0, & \text { for } q>r-1\end{cases}
$$

\subsection{General abelianization}

We may drop the nonresonance restriction from Theorem 5.3(2). We obtain the following (a priori, non-combinatorial) formula for the rank of an arbitrary character-abelianization, in terms of the fundamental group and the Euler characteristic.

THEOREM 5.6. - Let $\mathcal{A}$ be an essential arrangement in $\mathrm{P}^{r-1}$, where $r \geqslant 3$. Assume that $\mathcal{A}$ is a proper, $\mathcal{L}_{r-1}(\widehat{\mathcal{A}})$-generic section of an essential aspherical arrangement, $\widehat{\mathcal{A}}$. Set $M:=M(\mathcal{A})$ and $\pi:=\pi_{1}(M)$. Let $\nu: \pi \rightarrow \mathbb{Z}$ be an arbitrary character. Denote by $R:=\mathbb{K} \mathbb{Z}$ the associated local system on $M$, where $\mathbb{K}$ is any commutative field. Then:

$$
\operatorname{rank}_{R} \pi_{r-1}(M(\mathcal{A})) \otimes_{\nu} R=(-1)^{r-1}\left[\chi(M(\mathcal{A}))-\sum_{q=0}^{r}(-1)^{q} \operatorname{rank}_{R} \operatorname{Tor}_{q}^{\mathbb{Z} \pi}(\mathbb{Z}, R)\right]
$$

Proof. - The homotopy formula (4.1) says that $Y^{(r-1)}$ is a minimal $C W$-structure on $M(\mathcal{A})$, where $Y$ is a minimal structure on $M(\widehat{\mathcal{A}})$.

We are going to use the Künneth spectral sequence from [19, Theorem XII.12.1], arising from the free right $\mathbb{Z} \pi$-chain complex, $C_{\bullet}\left(\widetilde{Y}^{(r-1)}\right)$, and the change of rings map, $\nu: \mathbb{Z} \pi \rightarrow R$.

The $E^{2}$-term, $E_{s t}^{2}=\operatorname{Tor}_{s}^{\mathbb{Z} \pi}\left(H_{t} \tilde{Y}^{(r-1)}, R\right)$, is possibly nonzero only for $t=0$ (where $\left.H_{t} \widetilde{Y}^{(r-1)}=\mathbb{Z}\right)$, and for $t=r-1\left(\right.$ where $\left.H_{t} \widetilde{Y}^{(r-1)}=\pi_{r-1}:=\pi_{r-1}(M)\right)$. Hence, $E^{2}=E^{r}$, and $E^{r+1}=E^{\infty}$.

Since the spectral sequence converges to $H_{s+t}(M ; R)$, and $H_{r}(M ; R)=0$, we infer that

$$
d^{r}: E_{r 0}^{r}=\operatorname{Tor}_{r}^{\mathbb{Z} \pi}(\mathbb{Z}, R) \hookrightarrow \pi_{r-1} \otimes_{\nu} R=E_{0, r-1}^{r}
$$


is monic. Convergence also provides the exact sequence

$$
0 \rightarrow E_{0, r-1}^{\infty}=\operatorname{coker}\left\{d^{r}\right\} \rightarrow H_{r-1}(M ; R) \rightarrow E_{r-1,0}^{\infty}=\operatorname{Tor}_{r-1}^{\mathbb{Z} \pi}(\mathbb{Z}, R) \rightarrow 0 .
$$

Looking at $R$-ranks in (5.9), and also taking into account (5.8), we find that

$$
\operatorname{rank}_{R} \pi_{r-1} \otimes_{\nu} R=\operatorname{rank}_{R} H_{r-1}(M ; R)-\operatorname{rank}_{R} \operatorname{Tor}_{r-1}^{\mathbb{Z} \pi}(\mathbb{Z}, R)+\operatorname{rank}_{R} \operatorname{Tor}_{r}^{\mathbb{Z} \pi}(\mathbb{Z}, R) .
$$

We may now substitute in Eq. (5.10) above $\operatorname{rank}_{R} H_{r-1}(M(\mathcal{A}) ; R)$ by the formula from Theorem 4.5(1), to get the asserted formula for $\operatorname{rank}_{R} \pi_{r-1}(M(\mathcal{A})) \otimes_{\nu} R$.

The $R$-modules, $\pi_{r-1}(M(\mathcal{A})) \otimes_{\nu} R$ from Theorem 5.6, and $H_{r-1}(M(\mathcal{A}) ; R)$ from Theorem 4.5(1), look similar. Indeed, $\pi_{r-1}(M(\mathcal{A})) \otimes_{\nu} R=H_{r-1}\left(C_{\bullet}\right) \otimes_{\mathbb{Z} \pi} R$, and $H_{r-1}(M(\mathcal{A})$; $R)=H_{r-1}\left(C_{\bullet} \otimes_{\mathbb{Z} \pi} R\right)$, where $C_{\bullet}:=C_{\bullet}\left(\widetilde{Y}^{(r-1)}\right)$, and $Y$ is a minimal structure on $M(\widehat{\mathcal{A}})$. In fact, they turn out to be different, in general, even at the level of $R$-ranks.

EXAMPLE 5.7. - Let $\widehat{\mathcal{A}}$ be the essential arrangement in $\mathbb{P}^{r}$ associated to the braid arrangement from $\mathbb{P}^{r+1}, r \geqslant 3$. Let $\mathcal{A}$ be a generic (i.e., $\mathcal{L}_{r-1}(\widehat{\mathcal{A}})$-generic) hyperplane section of $\widehat{\mathcal{A}}$. Let $\pi$ be the pure braid group on $r+2$ strings $\left(\pi=\pi_{1}(M(\mathcal{A}))=\pi_{1}(M(\widehat{\mathcal{A}}))\right)$. Let $\nu: \pi \rightarrow \mathbb{Z}$ be $\widehat{\mathcal{A}}$-nonresonant, giving rise to the local system $R:=\mathbb{C} \mathbb{Z}$.

Then $\operatorname{rank}_{R} \pi_{r-1}(M(\mathcal{A})) \otimes_{\nu} R=\operatorname{rank}_{R} H_{r-1}(M(\mathcal{A}) ; R)$ if and only if

$$
\operatorname{rank}_{R} H_{r-1}(Y ; R)=\operatorname{rank}_{R} H_{r}(Y ; R),
$$

where $Y$ is a minimal structure on $M(\widehat{\mathcal{A}})$; see Theorem 5.6 and Theorem 4.5(1).

By Remark 5.4, (5.11) holds if and only if $\chi(M(\widehat{\mathcal{A}}))=0$. This in turn is impossible, since the well-known Poincaré polynomial of $M(\widehat{\mathcal{A}})$ is $\prod_{d=2}^{r+1}(1+d T)$.

\section{Acknowledgement}

This work was completed while the second author was visiting the University of Bordeaux. He thanks the CNRS and the Bordeaux Department of Mathematics for the excellent working facilities provided.

\section{REFERENCES}

[1] Cohen D., Cohomology and intersection cohomology of complex hyperplane arrangements, $A d v$. Math. 97 (1993) 231-266.

[2] Cohen D., Dimca A., ORLiK P., Nonresonance conditions for arrangements, Annales Institut Fourier 53 (2003) 1883-1896.

[3] Cohen D., ORlik P., Arrangements and local systems, Math. Res. Lett. 7 (2000) 299-316.

[4] Cohen D., Suciu A., On Milnor fibrations of arrangements, J. London Math. Soc. 51 (1995) 105119.

[5] Cohen D., Suciu A., The braid monodromy of plane algebraic curves and hyperplane arrangements, Comment. Math. Helv. 72 (1997) 285-315.

[6] Cohen D., Suciu A., Homology of iterated semidirect products of free groups, J. Pure Appl. Algebra 126 (1998) 87-120.

[7] Dimca A., NÉMEThi A., Hypersurface complements, Alexander modules and monodromy, preprint math.AG/0201291.

[8] Dimca A., PAPAdima S., Hypersurface complements, Milnor fibers and higher homotopy groups of arrangements, Ann. Math. 158 (2003) 473-507. 
[9] EIsenbud D., Commutative Algebra with a View Toward Algebraic Geometry, in: Grad. Texts in Math., vol. 150, Springer-Verlag, New York, 1995.

[10] FALK M., RANDELL R., The lower central series of a fiber-type arrangement, Invent. Math. 82 (1985) 77-88.

[11] Gibson C.G., Wirthmüller K., DU Plessis A.A., Looijenga E.J.N., Topological Stability of Smooth Mappings, in: Lecture Notes in Math., vol. 552, Springer-Verlag, Berlin, 1976.

[12] Goresky M., MacPherson R., Stratified Morse Theory, in: Ergebnisse, vol. 14, Springer-Verlag, New York, 1988.

[13] HATTORI A., Topology of $C^{n}$ minus a finite number of affine hyperplanes in general position, J. Fac. Sci. Univ. Tokyo 22 (1975) 205-219.

[14] Hillman J.A., Alexander Ideals of Links, in: Lecture Notes in Math., vol. 895, Springer-Verlag, Berlin, 1981.

[15] JAMBu M., PAPAdima S., A generalization of fiber-type arrangements and a new deformation method, Topology 37 (1998) 1135-1164.

[16] Jambu M., PAPadima S., Deformations of hypersolvable arrangements, Topology Appl. 118 (2002) $103-111$.

[17] LibGober A., On the homotopy type of the complement to plane algebraic curves, J. Reine Angew. Math. 397 (1986) 103-114.

[18] Libgober A., Homotopy groups of the complements to singular hypersurfaces II, Ann. Math. 139 (1994) 117-144.

[19] Mac LANe S., Homology, in: Grundlehren, vol. 114, Springer-Verlag, Berlin, 1963.

[20] ORLik P., Solomon L., Combinatorics and topology of complements of hyperplanes, Invent. Math. 56 (1980) 167-189.

[21] ORlik P., Terao H., Arrangements of Hyperplanes, in: Grundlehren, vol. 300, Springer-Verlag, Berlin, 1992.

[22] Papadima S., Suciu A., Higher homotopy groups of complements of complex hyperplane arrangements, Adv. Math. 165 (2002) 71-100.

[23] Randell R., Morse theory, Milnor fibers and minimality of hyperplane arrangements, Proc. Amer. Math. Soc. 130 (2002) 2737-2743.

[24] RYBNikov G., On the fundamental group of the complement of a complex hyperplane arrangement, available at math.AG/9805056, DIMACS Tech. Report 94-13 (1994) 33-50.

[25] Schechtman V., Terao H., Varchenko A., Local systems over complements of hyperplanes and the Kac-Kazhdan condition for singular vectors, J. Pure Appl. Algebra 100 (1995) 93-102.

[26] WhiteheAd G.W., Elements of Homotopy Theory, in: Grad. Texts in Math., vol. 61, Springer-Verlag, New York, 1978.

\author{
Alexandru DiMCA \\ Laboratoire J.A. Dieudonné, \\ UMR du CNRS 6621, \\ Université de Nice-Sophia-Antipolis, \\ Parc Valrose, \\ 06108 Nice Cedex 02, France \\ E-mail: dimca@math.unice.fr \\ Ştefan PAPADIMA \\ Inst. of Math. "Simion Stoilow", \\ P.O. Box 1-764, \\ 014700 Bucharest, Romania \\ E-mail: Stefan.Papadima@imar.ro
}

